\title{
Measurements of edge density profile modifications during IBW on TFTR
}

\author{
G. R. Hanson, C. E. Bush, J. B. Wilgen, T. S. Bigelow \\ Oak Ridge National Laboratory, Oak Ridge, TN 37831-6006. \\ J. H. Rogers, J. R. Wilson \\ Plasma Physics Laboratory, Princeton University, Princeton, NJ 08544
}

\begin{abstract}
Ion Bernstein wave (IBW) antennas are known to have substantial localized effects on the plasma edge. To allow better understanding and measurement of these effects, the TFTR edge reflectometer has been relocated to the new IBW antenna. This move was facilitated by the incorporation of a diagnostic access tube in the IBW antenna identical to the original diagnostic tube in the fast-wave (FW) antenna. This allowed the reflectometer launcher to simply be moved from the old FW antenna to the new IBW antenna. Only a moderate extension of the waveguide transmission line was required to reconnect the reflectometer to the launcher in its new location. Edge density profile modification during IBW experiments has been observed. Results from IBW experiments will be presented and contrasted to the edge density modifications previously observed during FW heating experiments.
\end{abstract}

\section{INTRODUCTION}

As part of the new IBW experiments on TFTR, the edge reflectometer launcher has been moved from the fast-wave (FW) antenna located in Bay $\mathrm{K}$ to the new IBW antenna located in Bay N. The edge reflectometer has previously been very successful at measuring edge density profile modification in front of the FW antenna. ${ }^{1}$ In general, the modifications observed can be classified into two components: 1) a decrease in the density and a flattening of the density gradient in the private flux zone of the antenna, and 2) an increase in the edge density inside the last closed flux surface. The modification of the density gradient in the private flux zone of the antenna has shown a dependence on both RF power and current strap phasing. Additionally, these modifications in the private flux zone of the FW antenna are pronounced with high power RF, but they are occurring at very low densities and so have only a minor effect on the antenna loading. In contrast, the increase in density inside the last closed flux surface can have significant effects on the loading. ${ }^{2}$

The edge profile reflectometer is a differential-phase extraordinary mode system operating from $90-118 \mathrm{GHz}$ and is presently using an $80 \mathrm{MHz}$ difference frequency. To facilitate relocating the reflectometer to the new IBW antenna, the new antenna was built with a diagnostic access tube identical to that in the FW antenna. ${ }^{1}$ This allowed the reflectometer launcher to be removed from the old antenna and installed directly into the new antenna. The waveguide transmission line was then modified to reconnect to the launcher in its new location. This increased the round trip length of the waveguide transmission line from $26 \mathrm{~m}$ to approximately $40 \mathrm{~m}$ with a corresponding increase in the round trip insertion loss

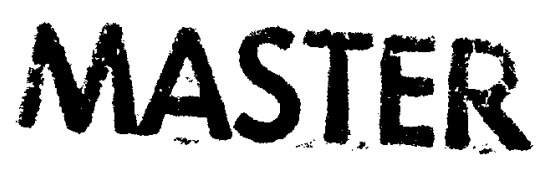

DISTFIBUTION OF THIS DOCUMENT IS UNLIMHTED 


\section{DISCLAIMER}

This report was prepared as an account of work sponsored by an agency of the United States Government. Neither the United States Government nor any agency thereof, nor any of their employees, make any warranty, express or implied, or assumes any legal liability or responsibility for the accuracy, completeness, or usefulness of any information, apparatus, product, or process disclosed, or represents that its use would not infringe privately owned rights. Reference herein to any specific commercial product, process, or service by trade name, trademark, manufacturer, or otherwise does not necessarily constitute or imply its endorsement, recommendation, or favoring by the United States Government or any agency thereof. The views and opinions of authors expressed herein do not necessarily state or reflect those of the United States Government or any agency thereof. 


\section{DISCLAMMER}

Portions of this document may be illegible in electronic image products. Images are produced from the best available original document. 
from $\leq 12-\mathrm{dB}$ to $\leq 15-\mathrm{dB}$. Two additional E-plane miter bends were required, for a total of $6 \mathrm{H}$-plane and $4 \mathrm{E}$-plane miter bends in the oversized transmission system. As with the Bay $\mathrm{K}$ installation, the reflectometer launcher views the plasma through a penetration at the geometric center of the Faraday Shield (FS) with the launcher aperture located $5 \mathrm{~mm}$ behind the surface of the FS.

\section{IBW RESULTS}

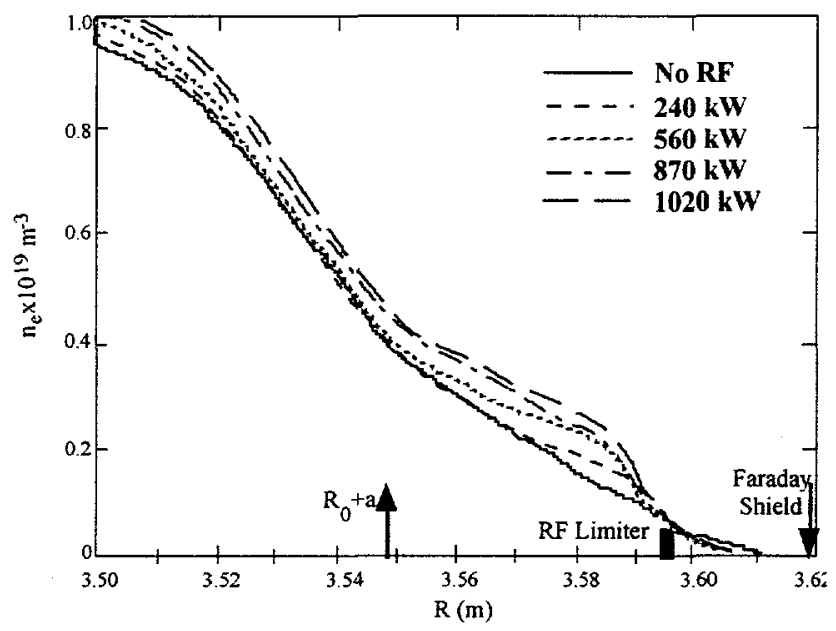

Figure 1. An IBW power scan at $4.74 \mathrm{~T}$ with $2.6 \mathrm{MW}$ neutral beam injection (NBI) heating.
Substantial modification of the edge density gradient has been observed during IBW heating. The typical effect, as shown in Fig. 1, consists of a drop in the plasma density in the private flux zone of the IBW antenna and a global increase in the density inside of the $\mathrm{RF}$ limiter radius. As seen in Fig. 1, the IBW power creates a low density region immediately in front of the FS. This region is followed by a steep gradient leading to the region of increased density. One interesting observation from this power scan is the creation of a 'bump' in the density gradient just inside of the RF limiter, e.g. the density increase just inside the limiter is larger than the increase inside of the LCF surface (as denoted by $\mathrm{R}_{0}+\mathrm{a}$ ). The density 'bump' increases in size with increasing IBW power. The apparent effect is consistent with a pushing away of the plasma (possibly by ponderomotive forces) immediately in front of the FS in the private zone of the antenna, but with no apparent variation in the size of the perturbation with increasing RF power, at the high power levels shown in Fig. 1.

Figure 2 shows the edge profile modification for IBW power levels of 1, 5 and $5.5 \mathrm{~kW}$. Note that with $1 \mathrm{~kW}$ of IBW power some modification of the profile in the private zone is visible. The small modification is at or below the typical accuracy of these measurements, but the measured differential phase data (not shown) clearly shows a change between the two profiles. The $5 \mathrm{~kW}$ and $5.5 \mathrm{~kW}$ cases show substantial modification in the private zone. These three cases are from three sequential shots with fairly reproducible plasma conditions. In Fig. 3, the modification due to $6 \mathrm{~kW}$ of IBW power is compared to the modification due to $1 \mathrm{MW}$ of power from two sequential shots. Note that the modification in the private zone is not substantially larger for $1 \mathrm{MW}$ than for $6 \mathrm{~kW}$, while the density 
increase inside of the RF limiter grows substantially at higher power. Figures 2 and 3 demonstrate that the observed modification in the private zone appears to occur predominantly in the first $\sim 6 \mathrm{~kW}$, while Fig. 1 shows that at higher power levels, the modification in the private zone is essentially constant with increasing power. So it appears that for the most

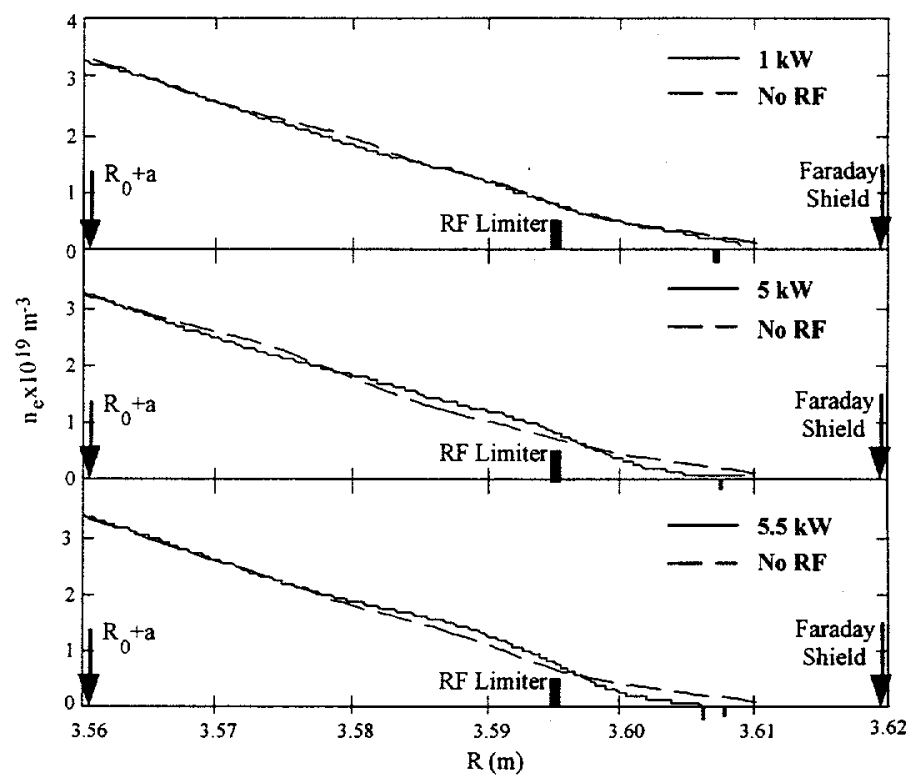

Figure 2. Comparison of the edge profiles for $1,5 \& 5.5 \mathrm{~kW}$ of IBW power in a $4.74 \mathrm{~T}$ plasma with $2.6 \mathrm{MW}$ of NBI. part, the modification of the density profile in the private zone of the antenna essentially saturates in the ten kilowatt range. This observation also agrees with the observed changes in the IBW antenna loading, which is predominantly dependent on the density profile at densities below $5 \times 10^{12} \mathrm{~cm}^{-3}$. The loading changes significantly in the first few kilowatts of applied IBW power, but is then essentially constant all the way up to $1 \mathrm{MW}$.

An important issue with this data is the accuracy of profile shapes (gradients) and the accuracy of the radial location of the profiles. The differentialphase measurement of the reflectometer directly measures the density gradient. Substantial averaging of the measured phase data is used in the data shown here to provide the best possible time-averaged representation of the profiles. Therefore, errors in the measured gradients are believed to be small compared to the size of the perturbations being studied here. Of greater concern is the accuracy to which the profiles are located radially. In reconstructing the density profile from the measured differential-phase, a starting location is assumed and the profile then reconstructed one step at a time to form the profile. The starting point for the profile reconstruction is constrained to be somewhere between the reflectometer launcher radius (the Faraday shield radius in this case) and the electron cyclotron radius (ECR) corresponding to the lowest frequency in the measured differential phase. For these data, this provides a range of typically 10 to $15 \mathrm{~mm}$. This is substantially larger than the effects being shown in the data and so additional details about the data are being used to more accurately locate the profiles. For example, the amplitude of the reflected reflectometer signal is very low in the first few hundred megahertz of signal. This is consistent with very low density, e.g. in 


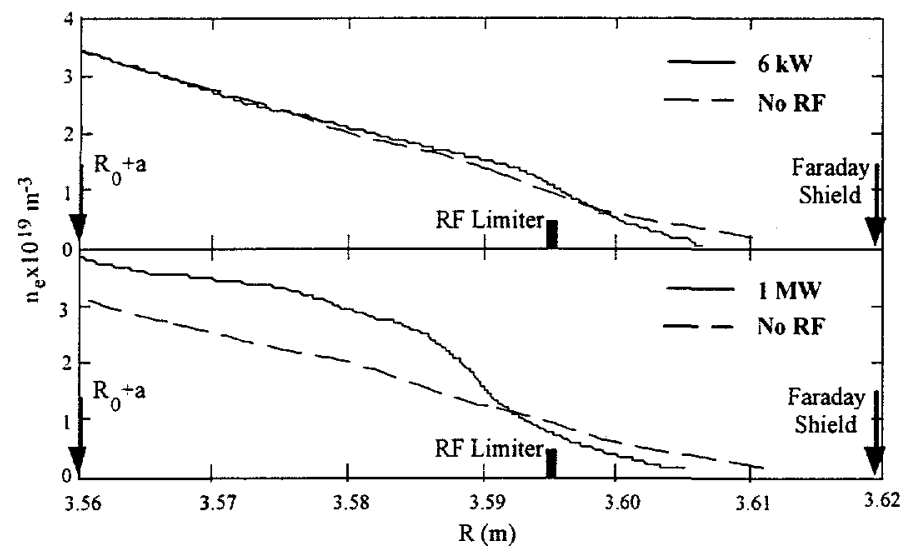

Figure 3. Comparison of the edge modification by $6 \mathrm{~kW}$ and $1 \mathrm{MW}$ of IBW power in a $4.87 \mathrm{~T}$ plasma with $2.6 \mathrm{MW}$ of NBI the low $10^{11} \mathrm{~cm}^{-3}$ or less, where only a portion of the signal is reflected. This forces the starting point to be moved to within $5 \mathrm{~mm}$ or less of the ECR. Additionally, if the phase data is starting at the same frequency and has the same values at the beginning, it is very probable that the initial

part of the profiles are the same. This is the case for the four IBW profiles shown in Fig. 1. They all have the same starting frequency and phase data for the first couple of gigahertz of frequency sweep; therefore indicating that the profiles overlap at their beginning location. The relative location of the with-RF to the noRF profiles is not as simple. In these cases, if the FIR interferometer data shows constant line-densities between the times of the two profiles, then it is assumed that the reflectometer profiles must overlap inside of the last closed flux surface.

\section{CONCLUSIONS}

Modification of the edge density profile by the Bay N IBW antenna has been observed and is qualitatively similar to the modifications previously observed at the Bay K FW antenna. IBW driven modifications differ from the FW driven modifications in that the density gradient is shortened near the RF limiter to form a bump (localized increase) in the density profile between the RF limiter and the last closed flux surface. Both IBW and Fast Wave density modifications show a dependence between the applied RF power and the size of the density modification, although the IBW driven modifications in the private zone of the antenna seem to saturate with the first $10 \mathrm{~kW}$ of applied power.

\section{ACKNOWLEDGMENTS}

The authors would like to thank Stan Milora and Dan Hoffman of ORNL and Joel Hosea and Randy Wilson of PPPL for their many years of support and encouragement of this project. Research sponsored by the Office of Fusion Energy, U.S. Department of Energy, under contract DE-AC05-96OR22464 with Lockheed Martin Energy Research Corporation.

\section{REFERENCES}

'Hanson, G. R. et al., Plasma Phys. Control. Fusion 36, 2073 (1994).

${ }^{2}$ Hanson, G. R. et al., "Edge Density Modification with rf on TFTR and DIII-D," 11 th Topical Conference on RF Power in Plasmas, Palm Springs, CA, 1995 (American Institute of Physics, New York, 1992) AIP Conf. Proc. 355 (1996) p. 463. 